(C) 2017, Elsevier. Licensed under the Creative Commons AttributionNonCommercial-NoDerivatives 4.0 International

http://creativecommons.org/licenses/by-nc-nd/4.0/

the Neurological Sciences

Elsevier Editorial System(tm) for Journal of Manuscript Draft

Manuscript Number: JNS-D-16-01956R2

Title: Malignancy in Guillain-Barré syndrome: a twelve-year singlecenter study

Article Type: Research paper

Keywords: axonal; cancer; Guillain-Barré syndrome; hyponatremia; malignancy; paraneoplastic.

Corresponding Author: Professor Yusuf A. Rajabally, M.D. F.R.C.P

Corresponding Author's Institution: University Hospitals of Birmingham

First Author: Fu Liong Hiew, MD

Order of Authors: Fu Liong Hiew, MD; Yusuf A. Rajabally, M.D. F.R.C.P

Abstract: The relationship between Guillain-Barré syndrome (GBS) and malignancy is uncertain.

We retrospectively analyzed data of 118 consecutive patients admitted with GBS from Birmingham, U.K. (2001-2012). We calculated relative cancer risk using different definitions and determined characteristics of malignancy-associated GBS. Malignancy was globally commoner in our GBS cohort compared to the general population (Odds Ratio: 2.08; CI: 1.06$3.71 ; \mathrm{p}=0.036)$. However this was unconfirmed if paraneoplastic criteria were applied. GBS patients with cancer were significantly more likely to be older $(p=0.043)$, hyponatremic $(p=0.037)$ and demonstrate more axonal loss $(p<0.05)$. Cerebrospinal fluid (CSF) protein levels were lower in the malignancy group $(\mathrm{p}=0.002)$ and neurological improvement less likely $(\mathrm{p}=0.023)$. In-patient mortality was significantly higher in patients with malignancy $(p<0.01)$. We conclude global cancer risk is higher in GBS than in the general population, although definition-dependent. Malignancy requires consideration in elderly, hyponatremic subjects with normal CSF protein, severe axonal loss, who fail to improve post-treatment. 
06.02.2017.

Dear Prof. England,

Thank you for allowing us to revise this paper.

We have altered the manuscript and Table in line with Reviewer 1's comments.

We are submitting 2 versions of this revised manuscript $\mathrm{R} 2$ with the changes in red ink in one.

We hope this paper will now be acceptable for publication in the Journal of Neurological Sciences.

With many thanks for your consideration.

Best wishes.

Yusuf Rajabally. 


\section{*Detailed Response to Reviewers}

-Reviewer 1:

We have acknowledged the limitation of our study due to its retrospective design and relatively small numbers studied, in these words in the text and also mention that cautious interpretation is therefore needed.

We have added the range of ages to mean and S.D., in Table 1.

We hope all changes, in red ink in the marked copy, will be acceptable.

Reviewer 2:

We thank this Reviewer for accepting the paper. 


\title{
Malignancy in Guillain-Barré syndrome: a twelve-year single-center study
}

\author{
Fu Liong Hiew, ${ }^{1}$ \\ Yusuf A. Rajabally. ${ }^{1,2}$
}

Regional Neuromuscular Clinic, Queen Elizabeth Hospital, University Hospitals of Birmingham, Birmingham, United Kingdom. ${ }^{1}$

School of Life and Health Sciences, Aston Brain Centre, Aston University, Birmingham, United Kingdom. ${ }^{2}$

Highlights:

-The link between malignancy and Guillain-Barré syndrome is uncertain.

-We found a higher malignancy risk in a Guillain-Barré syndrome cohort analyzed retrospectively over 12 years

-The malignancy risk in Guillain-Barré syndrome is dependent upon definitions used

-Malignancy risk in Guillain-Barré syndrome is greater in older, hyponatremic subjects with greater axonal loss and lower cerebrospinal fluid protein levels 


\title{
Malignancy in Guillain-Barré syndrome: a twelve-year single-center study
}

\author{
Fu Liong Hiew, ${ }^{1} \quad$ Yusuf A. Rajabally. ${ }^{1,2}$
}

Regional Neuromuscular Clinic, Queen Elizabeth Hospital, University Hospitals of Birmingham, Birmingham, United Kingdom. ${ }^{1}$

School of Life and Health Sciences, Aston Brain Centre, Aston University, Birmingham, United Kingdom. ${ }^{2}$

Revised Version R2.

Abstract Word Count: 150

Word Count: 2853

Number of Tables: 1

Number of Figures: 0

Number of References: 21

Funding: None

Abbreviations: AIDP: acute inflammatory demyelinating polyneuropathy; GBS:

Guillain-Barré syndrome

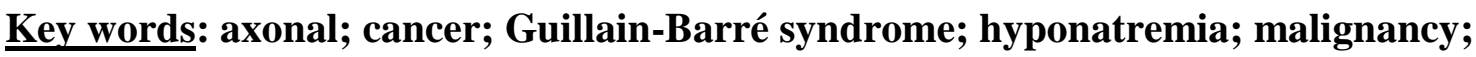
paraneoplastic.

Acknowledgement: We thank Dr. Peter Nightinghale, Statistician, University Hospitals of Birmingham, Birmingham, U.K., for the statistical analyses.

Funding: None.

Highlights:

-The link between malignancy and Guillain-Barré syndrome is uncertain.

-We found a higher malignancy risk in a Guillain-Barré syndrome cohort analyzed retrospectively over 12 years

-The malignancy risk in Guillain-Barré syndrome is dependent upon definitions used -Malignancy risk in Guillain-Barré syndrome is greater in older, hyponatremic subjects with greater axonal loss and lower cerebrospinal fluid protein levels

Correspondence to:

Yusuf A. Rajabally

School of Life \& Health Sciences,

Aston Brain Centre,

Aston University,

Aston Triangle,

Birmingham B4 7ET,

U.K.

E-mail: y.rajabally@aston.ac.uk 


\begin{abstract}
:
The relationship between Guillain-Barré syndrome (GBS) and malignancy is uncertain. We retrospectively analyzed data of 118 consecutive patients admitted with GBS from Birmingham, U.K. (2001-2012). We calculated relative cancer risk using different definitions and determined characteristics of malignancy-associated GBS. Malignancy was globally commoner in our GBS cohort compared to the general population (Odds Ratio: 2.08; CI: 1.06-3.71; $\mathrm{p}=0.036)$. However, this was unconfirmed if paraneoplastic criteria were applied. GBS patients with cancer were significantly more likely to be older $(\mathrm{p}=0.043)$, hyponatremic $(\mathrm{p}=0.037)$ and demonstrate more axonal loss $(\mathrm{p}<0.05)$. Cerebrospinal fluid $(\mathrm{CSF})$ protein levels were lower in the malignancy group $(\mathrm{p}=0.002)$ and neurological improvement less likely ( $\mathrm{p}=0.023)$. In-patient mortality was significantly higher in patients with malignancy $(\mathrm{p}<0.01)$. We conclude global cancer risk is higher in GBS than in the general population, although definition-dependent. Malignancy requires consideration in elderly, hyponatremic subjects with normal CSF protein, severe axonal loss, who fail to improve post-treatment.
\end{abstract}




\section{Introduction.}

\section{Guillain-Barré syndrome (GBS) is an acute, frequently post-infective} polyradiculoneuropathy, occurring after gastrointestinal or respiratory infections. There is considerable literature consisting mostly of case reports of GBS described in the context of malignancies, although the presence of a definite association is unproven. The association of malignancy and GBS without evidence of direct tumor infiltration, was described over 50 years ago, by Klingon who postulated that the co-occurrence may represent an immune response of the peripheral nervous system [1]. However, a common immunopathogenesis between GBS and cancer has not to date been demonstrated and no specific onconeural antibodies have been identified. To our knowledge, a single population-based study has been conducted evaluating the risk of cancer in GBS patients [2]. This analysis from Italy described an estimated moderately increased risk of malignancy in GBS, with an odds ratio of 2.37 to 2.43 . The characteristics of cancer-associated GBS remain uncertain.

Following the recent development in the concept of paraneoplastic peripheral nervous system disorders, neuronopathy/neuropathy have been recognized as paraneoplastic manifestation in relation to the occurrence of underlying malignancy. In most cases, paraneoplastic neurological syndromes can occur months or years before the diagnosis of cancer with detection of onconeural antibodies directed against neural antigens expressed by the tumor [3]. The definition and diagnostic criteria for paraneoplastic peripheral nervous system disorders was proposed by the Paraneoplastic Neurological Syndrome Euronetwork in 2004 [4]. GBS has been classified as "non-classical paraneoplastic PNS disorder", in contrast to subacute sensory neuronopathy, included as part of the "classical paraneoplastic" disorders. With no definite onconeural antibodies identified, diagnosis of GBS in a patient with known 
cancer classifies as definitely paraneoplastic if it resolves or significantly improves after overwhelming majority of patients with GBS are in practice treated with immunotherapy before completion of any cancer treatment. The definition of cancer-associated GBS can clearly be variable and whether this may impact upon the frequency of the co-existence of the 2 disorders, appears possible.

The objectives of our study were firstly to retrospectively determine the frequency of cooccurrence of malignancy in a cohort of patients admitted to our tertiary hospital for GBS over a twelve-year period. We aimed to estimate the relative cancer risk in this population as well as evaluate the impact of different definitions for cancer-associated GBS. We in addition, planned to ascertain the differences in clinical characteristics and outcome between GBS patients with and without malignancy, aiming to establish which patients are most at risk of associated cancer and therefore likely to benefit from further investigations and extended follow-up. 


\section{Materials and methods}

We retrospectively reviewed our institutional data-base for GBS patients admitted between 2001 and 2012 to our in-patient unit at University Hospitals of Birmingham, United Kingdom. Patients with Miller Fisher syndrome (MFS) and subsequently confirmed acuteonset chronic inflammatory demyelinating polyneuropathy (CIDP) were excluded. Patients with incomplete clinical data were also excluded. This study was part of a wider retrospective audit on GBS, registered and approved at our institution (CAD- 05169-13, April 2013).

We defined GBS as a clinical diagnosis applying recently proposed diagnostic clinical criteria [5]. We classified patients in 2 groups, GBS with malignancy and GBS without malignancy. For those with malignancy, we determined the onset of GBS in relation to the diagnosis of malignancy. We utilized 2 different definitions for cancer-associated GBS. We first considered all cases of cancer diagnosed in the cohort, excluding preceding cancer diagnoses $>1$ year before GBS diagnosis and with no evidence of malignant disease activity at the time of GBS diagnosis. Secondly, we also in addition, excluded myelomas and malignancies diagnosed $>2$ years post-GBS onset as per paraneoplastic peripheral nervous system disorder criteria [4].

Patient demographics, duration of inpatient stay, intensive care unit admission, mechanical ventilation, Medical Research Council (MRC) sum score (MRCSS) at admission and discharge, treatment administered, were ascertained. For patients with cancer, the type of malignancy, timing of cancer diagnosis, malignancy status at time of GBS diagnosis, treatment administered for GBS, were ascertained. Electrophysiological data as well as 
biological and immunological data were reviewed. We determined the various features observed during admission and the clinical outcome of the patients with and without cancer. 


\section{Results.}

We identified a total of 118 patients admitted to our institution between 2001 and 2012 with a clinical diagnosis of GBS. Amongst these, 9 patients were excluded from the analysis due to incomplete data. A total of 12 patients were found to have malignancy. Of those 2 patients were immediately excluded, both with breast cancer, who had received this diagnosis 7 and 20 years, respectively, prior to their GBS and who displayed no evidence of cancer activity or progression at the time of the GBS diagnosis. This left an initial total number of associated cancers of 10 (9.17\% of the cohort).

Considering current definitions of paraneoplastic syndrome [4], we excluded a further 2 patients who had IgG paraprotein myeloma and an additional 2 patients who had malignancy diagnosed $>2$ years after the GBS presentation. One had prostatic carcinoma and the other a basal cell carcinoma, both 4 years after GBS.

Consequently, a total of 6 patients (5.5\% of our total cohort) fulfilled requirements for inclusion as malignancy-associated GBS as per existing criteria for paraneoplastic syndrome [4]. Amongst these, 3 patients developed malignancy prior to the GBS presentation. All of them had developed GBS while undergoing treatment for their underlying cancer.

Chemotherapy was not implicated in the development of neuropathy in any of these 3 patients. Two patients were diagnosed with cancer during their GBS presentation. One patient developed cancer one year after the GBS diagnosis. Of the 6 patients whose GBS fulfilled criteria for paraneoplastic neurological syndrome, there was one case of angioimmunoblastic T-cell lymphoma, one of poorly differentiated squamous cell carcinoma of the nasal septum, one of gastric adenocarcinoma, one of hepatocellular carcinoma due to hepatitis B, one of rectal carcinoma with liver metastasis and one case of myelodysplastic syndrome. 
Considering U.K. cancer incidence rates [6], as well as the number of patient years of followup taking into account timing of death, the expected cumulative cancer rate in this GBS cohort of 109 patients over the 15-year study period, including the 3 years after admission of the last recruited patient (December 2015), was of 4.80 patients. With 10 cancer cases considered, the odds ratio was 2.08 (95\% CI: 1.06-3.71) and therefore significantly higher than expected $(\mathrm{p}=0.036)$. However, considering only the 6 cases meeting the definition for paraneoplastic syndrome [4], there was no increased risk of cancer in GBS patients over the study period (standardized odds ratio: $1.25 ; 95 \%$ CI: 0.51-2.60; $\mathrm{p}=0.559$ ).

We analyzed our results considering the 6 cancer cases fulfilling criteria for paraneoplastic syndrome. These are summarized in Table 1. Of the 6 patients meeting this definition, 5 were males and 1 was female as compared to 70 males and 33 females in the non-malignancy group ( $\mathrm{p}=0.66$ ). Average age in the malignancy group was higher compared to the nonmalignancy group (mean of 65.8 [S.D.:13.3] vs. 51.3 years [S.D.:17.5]; $p=0.043$ ). None of the patients in the malignancy group had positive anti-ganglioside antibodies or antineuronal antibodies (anti-Hu, Yo, Ri, CRMP5). There was no difference of lowest forced vital capacity (FVC) recorded among both groups $(\mathrm{p}=0.78)$. Cerebrospinal fluid (CSF) studies were acellular in all cases in both groups. CSF protein levels were significantly lower in patients with cancer than in those without cancer (mean: $0.34 \mathrm{~g} / \mathrm{dL}$ [S.D.: 0.59] vs. mean: $1.37 \mathrm{~g} / \mathrm{dL}$ [S.D.: 1.21]; $\mathrm{p}=0.002)$. Hyponatremia was significantly more common amongst patients with malignancy (66.7\% vs. $23.3 \%$; $\mathrm{p}=0.037)$. A higher proportion $(3 / 6 ; 50 \%)$ of the patients in the malignancy group required ICU admission with mechanical ventilation compared to of those from the non-malignancy group $(15 / 103 ; 14.6 \%)$, this approaching significance $(p=0.055)$. Average length of stay was comparable in both groups (mean of 42.8 days vs. 23.6 days; $\mathrm{p}=0.20$ ). Although admission MRCSS were comparable in malignancy and non- 
malignancy groups), improvement during in-patient stay, defined as amelioration of admission MRCSS (Medical Research Council Sum Score) was significantly less frequent in the malignancy group $(\mathrm{p}=0.023)$. From the point of view of electrophysiological subtypes, 3 patients had acute inflammatory demyelinating polyradiculoneuropathy (AIDP) and 3 had axonal GBS, as categorized by new electrodiagnostic criteria [7]. There was no difference in the number of patients with different subtypes of GBS in both groups ( $p=1$ for AIDP and $\mathrm{p}=0.66$ for axonal GBS). Summated compound muscle action potential (CMAP) was significantly lower in the malignancy group (mean: $14.5 \mathrm{mV}$, S.D.: 10.7 vs. mean: $7.1 \mathrm{mV}$, S.D.: 7.0; $\mathrm{p}=0.0497)$. There was no difference in terms of treatment given to patients in both groups with the majority (100\% and $91.3 \%$ from malignancy and non-malignancy groups, respectively), treated with intravenous immunoglobulins. Only 2 of 6 patients (33.3\%) with malignancy were able to walk at discharge, comparing to 73 of 103 patients (70.9\%) without malignancy ( $\mathrm{p}=0.075$ ). In-patient mortality was significantly higher in the malignancy group compared to the non-malignancy group $(33.3 \%$ vs. $1 \% ; \mathrm{p}=0.0075)$.

Considering all 10 cancer cases, the analysis produced similar statistical results with significance however not reached for more frequent hyponatremia in patients with malignancy $(\mathrm{p}=0.12)$ nor for MRC grade improvement during in-patient stay $(\mathrm{p}=0.10)$. 


\section{Discussion.}

The relationship between GBS and malignancy has been a topic of controversy since its initial descriptions. An earlier report described 2 patients with typical GBS occurring in association with Hodgkin's disease and oat-cell lung carcinoma, without evidence of malignant invasion [1]. The likelihood of cancer as a possible precipitating cause of GBS was raised, through an immune response towards the peripheral nervous system. Since then, many anecdotal reports relating GBS with various types of malignancy have been published. Cases described include malignancy of lung, bladder, blood, colon and skin $[2,8,9,10]$. One major difficulty with previous reports is that some cases appear clearly likely paraneoplastic from onset despite a GBS-like presentation, while others, differed with a classical clinical GBS presentation, with no associated features to suggest a paraneoplastic phenomenon, despite an eventually diagnosed associated malignancy, raising the question about a link between the 2. Our patients are not in the first case scenario as all were diagnosed with GBS and this remained the final diagnosis at discharge/death. The focus of our study has therefore been on the basis of the second eventuality, which is of direct relevance to clinical management of patients presenting with GBS.

Paraneoplastic neurological syndromes occur in less than $1 \%$ of patients with malignancy [3, 11]. Many cases present months to years before the diagnosis of cancer [3, 11]. Recent developments in concept of paraneoplastic syndrome has led to the inclusion of varieties of neuropathies other than the classical paraneoplastic syndromes. Criteria for diagnosis of paraneoplastic PNS disorder were adopted from Graus et al., 2004 [4]. Definite paraneoplastic disorders of PNS include a classic paraneoplastic syndrome associated with cancer and onconeural antibodies. Paraproteinemic neuropathies are not included as 
paraneoplastic manifestation, although exception to this rule may be made in the case of

POEMS syndrome, as it is defined [12]. GBS has been considered as one of the "nonclassical paraneoplastic neurological syndromes without onconeuronal antibodies" [13]. Since GBS is not a classical PNS and there are no identified onconeuronal antibodies linking it to a specific tumor, improvement of the neuropathy following treatment of the tumor is a major criterion for diagnosis confirmation. However, most cases would have been treated with immunotherapy during the course of illness making the definite diagnosis of paraneoplastic syndrome, impossible.

A single population-based study by Vigliani et al. has demonstrated a possible association between GBS and cancer [2]. However, the occurrence of GBS in this cohort of patients did not meet all the criteria for paraneoplastic syndrome, as were subsequently proposed by Graus et al. [4]. In this regard, Vigliani et al. included only malignancies developing or recurring 6 months before or after GBS diagnosis [2]. This excludes delayed cancer diagnoses, known to occur in some cases years after the neurological presentation. The definition used for the association is therefore of major importance in determining the existence of a link between the 2 disorders. We here used 2 definitions and confirm a variable risk, depending on which one is used. Although cancer in GBS, itself considered as a possible non-classical paraneoplastic syndrome [4] appears no more common than cancer in the general population, the total number of cancers does appear significantly higher in a cohort of patients with a GBS presentation. One reason may be that the definition for paraneoplastic neurological disease is too restrictive and wrongly excludes several relevant cases where there still may exist a causal relationship between the cancer and the neurological presentation, here GBS. 
The mechanisms of suspected paraneoplastic neuropathies without onconeuronal antibodies are unknown. Anti-ganglioside antibodies have been described in some of GBS patients with cancer, such as melanoma [14]. GBS encompasses a group of peripheral nerve disorders of autoimmune origin with, in axonal forms, evidence supporting the underlying pathophysiological mechanism involving the presence of molecular mimicry between gangliosides and antigens of an antecedent infection, stimulating a cross-reaction with peripheral nerve components [15]. Previous cases have been described of paraneoplastic motor neuropathy in patients with anti-GM1 ganglioside antibodies associated with epidermoid esophageal carcinoma [16], melanoma [10], bladder [8] and small cell lung carcinoma $[17,18]$. Whether the detection of anti-gangliosides antibodies may be relevant in patients with GBS with malignancy remains however uncertain. It was postulated that the expression of gangliosides in the neoplastic tissue may elicit autoimmune responses against neural structures [19]. In a case-control study comparing 29 patients with cancer and neuropathies with controls, anti-ganglioside antibodies, mainly IgM anti-GM1 were more frequently found in patients with cancer [20]. However, the pattern and levels of antibodies were not different from those of the controls and it appeared possible that the higher occurrence of anti-ganglioside antibodies in cancer patients may relate to a concurrent antitumor immune reaction rather than causative of the neuropathic process. The absence of antigangloside antibodies in all cases with cancer in our GBS cohort would similarly plead against their implication in the neuropathy. Despite absence of a definite antibody marker, it is possible that malignancy-induced immune dysregulation may be implicated in the pathogenesis of the acute inflammatory polyradiculoneuropathic process in patients with cancer and GBS. 
Our findings in relation to CSF protein, hyponatremia and electrophysiology are interesting. Elevated CSF protein levels are the expression of an inflammatory neural process, and it is possible that there may be pathophysiologic differences between the acute polyradiculoneuropathy of neoplastic disease and that of typical GBS explaining lower CSF protein in patients with malignancy. We unfortunately, in view of the retrospective nature of the analysis, do not however have the precise timings of lumbar punctures in many of our patients, some having been transferred from local district general hospitals to our center during their illness. We were therefore unable to ascertain whether patients with and without malignancy had lumbar punctures at comparable times. This is a drawback as early lumbar puncture may result in a higher likelihood of normal CSF protein levels. Hyponatremia was otherwise found more commonly in patients with malignancy as may have been expected, as not uncommon in cancer. In this regard, in view of the mild hyponatremia in the affected patients in the current analysis, further testing with serum and urine osmolality did not appear to have been performed. We previously found that hyponatremia is non-specific but a likely predictor of mortality in GBS [21]. Our current findings interestingly therefore suggest that hyponatremia may potentially increase suspicion of an underlying neoplasm in some patients with GBS. Finally, and similarly, more severe electrophysiological axonal loss was more frequent in patients affected by malignancy. Whether this may relate to associated preexisting asymptomatic neuropathy or to a more severe acute axonopathy, specifically occurring in these patients, is unknown.

In conclusion, our findings suggest that there may be an overall excess cancer risk in patients with a clinical diagnosis of GBS. Our results are in keeping with only the previous similar study performed [2]. The findings appear importantly also dependent on the definition used for cancer-associated GBS, and application of the strict published criteria for paraneoplastic 
syndrome reduced the number of cases and suggested absence of a link. We acknowledge that our study is limited by its retrospective design as well as relatively low numbers and that the findings therefore need to be considered with caution. However, these results may suggest importantly for clinical practice, that consideration of an associated, detectable or occult malignancy is warranted in some patients with a clinical presentation of GBS. This is particularly relevant in absence of a preceding infectious trigger in older age groups, in patients who do not improve as would be expected after treatment, as well as those with normal CSF protein, hyponatremia, and severe electrophysiological axonal loss. Our results finally raise the issue of adequate investigations which in such cases may justify early use of whole-body CT imaging as well as PET. Furthermore, although the long-term monitoring of patients with GBS months to years after diagnosis is rare in clinical practice, this may require consideration with careful clinical re-assessment for adequate further investigations at regular intervals, in selected cases. 


\section{References.}

1. Klingon GH. The Guillain-Barré syndrome associated with cancer. Cancer 1965;18:157-63.

2. Vigliani MC, Magistrello M, Polo P, Mutani R, Chiò A. Risk of cancer in patients with Guillain-Barre syndrome (GBS). J Neurol 2004;251:321-326.

3. Rudnicki SA, Dalmau J. Paraneoplastic syndromes of the spinal cord, nerve, and muscle. Muscle Nerve 2000;23:1800-1818.

4. Graus F, Delattre JY, Antoine JC, Dalmau J, Giometto B, Grisold W, et al. Recommended diagnostic criteria for paraneoplastic neurological syndromes. J Neurol Neurosurg Psychiatry 2004;75:1135-1140.

5. Wakerley BR, Uncini A, Yuki N, GBS Classification Group. Guillain-Barré and Miller Fisher syndromes--new diagnostic classification. Nat. Rev. Neurol. 2014;10:537-544.

6. Cancer U.K. http://www.cancerresearchuk.org/health-professional/cancer-statistics July 2016.

7. Rajabally YA, Durand MC, Mitchell J, Orlikowski D, Nicolas G. Electrophysiological diagnosis of Guillain-Barré syndrome subtype: could a single study suffice? J. Neurol. Neurosurg. Psychiatry 2015;86:115-119.

8. Lagrange E, Veran O, Besson G. Pure motor relapsing Guillain-Barre's syndrome associated with anti-GM1 antibodies revealing urinary bladder cancer European Journal of Neurology 2007;17:e7.

9. Vatandoust S, Joshi R and Price TJ. Guillain-Barré syndrome in colorectal cancer. Asia-Pacific Journal of Clinical Oncology 2012;8:205-208.

10. Nicolae CD, Nicolae I. Antibodies against GM1 gangliosides associated with metastatic melanoma. Acta Dermatovenerol Croat. 2012;21:86-92. 
11. Darnell RB, Posner JB. Paraneoplastic syndromes involving the nervous system. N Engl J Med 2003;349:1543-1554.

12. Dispenzieri A. POEMS syndrome: update on diagnosis, risk-stratification, and management. Am J Hematol. 2015;90:951-962.

13. Rudnicki SA, Dalmau J. Paraneoplastic syndromes of the peripheral nerves. Curr Opin Neurol 2005;18:598-603.

14. Kloos L, Sillevis Smitt P, Ang CW, Kruit W, Stoter G. Paraneoplastic ophthalmoplegia and subacute motor axonal neuropathy associated with anti-GQ1b antibodies in a patient with malignant melanoma. J Neurol Neurosurg Psychiatry 2003;74:507-509.

15. Yuki N, Hartung HP. Guillain-Barré syndrome. N Engl J Med 2012;366:2294-2304.

16. Mostoufizadeh S, Souri M, de Seze J. A case of paraneoplastic demyelinating motor polyneuropathy. Case Rep Neurol 2012;4:71-76.

17. Watanuki S, Kinoshita K, Oda A, Kobayashi H, Satoh H, Tokuda Y. Occam's Razor or Hickam's dictum: a paraneoplastic or coincidental occurrence of lung cancer and Guillain-Barré syndrome. Inter Med 2014;53:1569-1573.

18. Itou T, Enomoto S, Makita Y, Enomoto H, Kuroda K, Kimura T, et al. A patient of sensorimotor neuropathy with small cell lung carcinoma and anti-GM1 antibody. Rinsho Shinkeigaku 2002;42:878-80.

19. De Toni L, Marconi S, Nardelli E, Alberti D, Borsellino G, Fracasso G, et al. Gangliosides act as onconeural antigens in paraneoplastic neuropathies. $\mathbf{J}$ Neuroimmunol 2004;156:178-187.

20. Antoine JC, Camdessanché JP, Ferraud K, Caudie C. Antiganglioside antibodies in paraneoplastic peripheral neuropathies. J Neurol Neurosurg Psychiatry. 2004;75:1765-1767. 
21. Hiew FL, Winer JB, Rajabally YA. Hyponatraemia in Guillain-Barré syndrome revisited. Acta Neurol Scand 2016;133:295-301. 
Table 1: Characteristics of 109 Guillain-Barré syndrome (GBS) patients, from Birmingham, U.K. (20012012) without and with malignancy as per paraneoplastic definitions [4].

\begin{tabular}{|l|l|l|l|}
\hline & GBS with malignancy & $\begin{array}{l}\text { GBS without } \\
\text { malignancy }\end{array}$ & $\begin{array}{l}\text { P value } \\
\text { (Fisher Exact } \\
\text { Test or T- } \\
\text { test) }\end{array}$ \\
\hline Number of patients & $6(5.5 \%)$ & $103(94.5 \%)$ & - \\
\hline Mean Age (SD; range) & $\begin{array}{l}65.8 \text { years (13.3; } 59- \\
87)\end{array}$ & 51.3 years (17.5) & $\mathbf{p}=\mathbf{0 . 0 4}$ \\
\hline Male: Female ratio & $5: 1$ & $70: 33$ & $\mathrm{p}=0.66$ \\
\hline AIDP Subtype & 3 & 47 & $\mathrm{p}=1$ \\
\hline Axonal GBS subtype & 3 & 36 & $\mathrm{p}=0.66$ \\
\hline Length of stay & 42.8 days & 23.6 days & $\mathrm{p}=0.20$ \\
\hline Hyponatremia & $4(66.7 \%)$ & $24(23.3 \%)$ & $\mathbf{p}=\mathbf{0 . 0 3 7}$ \\
\hline Average CSF protein mean (SD) & $0.34 \mathrm{~g} / \mathrm{L}(0.59)$ & $1.37 \mathrm{~g} / \mathrm{L}(1.21)$ & $\mathbf{p}=\mathbf{0 . 0 0 2}$ \\
\hline Lowest FVC & & & $\mathrm{p}$ \\
\hline ICU + ventilation & $2.61 \mathrm{I}$ & $2.87 \mathrm{I}$ & $\mathrm{p}=0.78$ \\
\hline Treated with immunoglobulins & $3(50 \%)$ & $15(14.6 \%)$ & $\mathrm{p}=0.055$ \\
\hline Average CMAP sum score & $6(100 \%)$ & $94(91.3 \%)$ & $\mathrm{p}=1$ \\
\hline $\begin{array}{l}\text { Number of patients with } \\
\text { Improvement in MRCSS }\end{array}$ & $7.1 \mathrm{mV}(7.0)$ & $14.5 \mathrm{mV} \mathrm{(10.7)}$ & $\mathbf{p}=\mathbf{0 . 0 4 9 7}$ \\
\hline $\begin{array}{l}\text { Number of patients able to walk at } \\
\text { discharge }\end{array}$ & 2 & 60 & $\mathbf{p}=\mathbf{0 . 0 2 3}$ \\
\hline In-patient mortality & $2(33.3 \%)$ & 73 & $\mathrm{p}=0.075$ \\
\hline
\end{tabular}




\title{
Malignancy in Guillain-Barré syndrome: a twelve-year single-center study
}

\author{
Fu Liong Hiew, ${ }^{1} \quad$ Yusuf A. Rajabally. ${ }^{1,2}$
}

Regional Neuromuscular Clinic, Queen Elizabeth Hospital, University Hospitals of Birmingham, Birmingham, United Kingdom. ${ }^{1}$

School of Life and Health Sciences, Aston Brain Centre, Aston University, Birmingham, United Kingdom. ${ }^{2}$

Revised Version R2.

Abstract Word Count: 150

Word Count: 2853

Number of Tables: 1

Number of Figures: 0

Number of References: 21

Funding: None

Abbreviations: AIDP: acute inflammatory demyelinating polyneuropathy; GBS:

Guillain-Barré syndrome

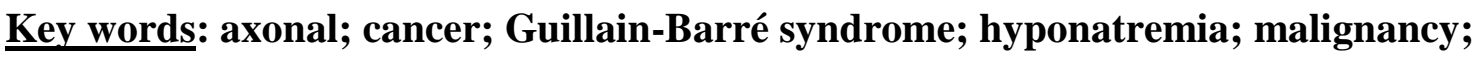
paraneoplastic.

Acknowledgement: We thank Dr. Peter Nightinghale, Statistician, University Hospitals of Birmingham, Birmingham, U.K., for the statistical analyses.

Funding: None.

Highlights:

-The link between malignancy and Guillain-Barré syndrome is uncertain.

-We found a higher malignancy risk in a Guillain-Barré syndrome cohort analyzed retrospectively over 12 years

-The malignancy risk in Guillain-Barré syndrome is dependent upon definitions used -Malignancy risk in Guillain-Barré syndrome is greater in older, hyponatremic subjects with greater axonal loss and lower cerebrospinal fluid protein levels

Correspondence to:

Yusuf A. Rajabally

School of Life \& Health Sciences,

Aston Brain Centre,

Aston University,

Aston Triangle,

Birmingham B4 7ET,

U.K.

E-mail: y.rajabally@aston.ac.uk 


\begin{abstract}
:
The relationship between Guillain-Barré syndrome (GBS) and malignancy is uncertain. We retrospectively analyzed data of 118 consecutive patients admitted with GBS from Birmingham, U.K. (2001-2012). We calculated relative cancer risk using different definitions and determined characteristics of malignancy-associated GBS. Malignancy was globally commoner in our GBS cohort compared to the general population (Odds Ratio: 2.08; CI: 1.06-3.71; $\mathrm{p}=0.036)$. However, this was unconfirmed if paraneoplastic criteria were applied. GBS patients with cancer were significantly more likely to be older $(\mathrm{p}=0.043)$, hyponatremic $(\mathrm{p}=0.037)$ and demonstrate more axonal loss $(\mathrm{p}<0.05)$. Cerebrospinal fluid $(\mathrm{CSF})$ protein levels were lower in the malignancy group $(\mathrm{p}=0.002)$ and neurological improvement less likely ( $\mathrm{p}=0.023)$. In-patient mortality was significantly higher in patients with malignancy $(\mathrm{p}<0.01)$. We conclude global cancer risk is higher in GBS than in the general population, although definition-dependent. Malignancy requires consideration in elderly, hyponatremic subjects with normal CSF protein, severe axonal loss, who fail to improve post-treatment.
\end{abstract}




\section{Introduction.}

\section{Guillain-Barré syndrome (GBS) is an acute, frequently post-infective} polyradiculoneuropathy, occurring after gastrointestinal or respiratory infections. There is considerable literature consisting mostly of case reports of GBS described in the context of malignancies, although the presence of a definite association is unproven. The association of malignancy and GBS without evidence of direct tumor infiltration, was described over 50 years ago, by Klingon who postulated that the co-occurrence may represent an immune response of the peripheral nervous system [1]. However, a common immunopathogenesis between GBS and cancer has not to date been demonstrated and no specific onconeural antibodies have been identified. To our knowledge, a single population-based study has been conducted evaluating the risk of cancer in GBS patients [2]. This analysis from Italy described an estimated moderately increased risk of malignancy in GBS, with an odds ratio of 2.37 to 2.43 . The characteristics of cancer-associated GBS remain uncertain.

Following the recent development in the concept of paraneoplastic peripheral nervous system disorders, neuronopathy/neuropathy have been recognized as paraneoplastic manifestation in relation to the occurrence of underlying malignancy. In most cases, paraneoplastic neurological syndromes can occur months or years before the diagnosis of cancer with detection of onconeural antibodies directed against neural antigens expressed by the tumor [3]. The definition and diagnostic criteria for paraneoplastic peripheral nervous system disorders was proposed by the Paraneoplastic Neurological Syndrome Euronetwork in 2004 [4]. GBS has been classified as "non-classical paraneoplastic PNS disorder", in contrast to subacute sensory neuronopathy, included as part of the "classical paraneoplastic" disorders. With no definite onconeural antibodies identified, diagnosis of GBS in a patient with known 
cancer classifies as definitely paraneoplastic if it resolves or significantly improves after overwhelming majority of patients with GBS are in practice treated with immunotherapy before completion of any cancer treatment. The definition of cancer-associated GBS can clearly be variable and whether this may impact upon the frequency of the co-existence of the 2 disorders, appears possible.

The objectives of our study were firstly to retrospectively determine the frequency of cooccurrence of malignancy in a cohort of patients admitted to our tertiary hospital for GBS over a twelve-year period. We aimed to estimate the relative cancer risk in this population as well as evaluate the impact of different definitions for cancer-associated GBS. We in addition, planned to ascertain the differences in clinical characteristics and outcome between GBS patients with and without malignancy, aiming to establish which patients are most at risk of associated cancer and therefore likely to benefit from further investigations and extended follow-up. 


\section{Materials and methods}

We retrospectively reviewed our institutional data-base for GBS patients admitted between 2001 and 2012 to our in-patient unit at University Hospitals of Birmingham, United Kingdom. Patients with Miller Fisher syndrome (MFS) and subsequently confirmed acuteonset chronic inflammatory demyelinating polyneuropathy (CIDP) were excluded. Patients with incomplete clinical data were also excluded. This study was part of a wider retrospective audit on GBS, registered and approved at our institution (CAD- 05169-13, April 2013).

We defined GBS as a clinical diagnosis applying recently proposed diagnostic clinical criteria [5]. We classified patients in 2 groups, GBS with malignancy and GBS without malignancy. For those with malignancy, we determined the onset of GBS in relation to the diagnosis of malignancy. We utilized 2 different definitions for cancer-associated GBS. We first considered all cases of cancer diagnosed in the cohort, excluding preceding cancer diagnoses $>1$ year before GBS diagnosis and with no evidence of malignant disease activity at the time of GBS diagnosis. Secondly, we also in addition, excluded myelomas and malignancies diagnosed $>2$ years post-GBS onset as per paraneoplastic peripheral nervous system disorder criteria [4].

Patient demographics, duration of inpatient stay, intensive care unit admission, mechanical ventilation, Medical Research Council (MRC) sum score (MRCSS) at admission and discharge, treatment administered, were ascertained. For patients with cancer, the type of malignancy, timing of cancer diagnosis, malignancy status at time of GBS diagnosis, treatment administered for GBS, were ascertained. Electrophysiological data as well as 
biological and immunological data were reviewed. We determined the various features observed during admission and the clinical outcome of the patients with and without cancer. 


\section{Results.}

We identified a total of 118 patients admitted to our institution between 2001 and 2012 with a clinical diagnosis of GBS. Amongst these, 9 patients were excluded from the analysis due to incomplete data. A total of 12 patients were found to have malignancy. Of those 2 patients were immediately excluded, both with breast cancer, who had received this diagnosis 7 and 20 years, respectively, prior to their GBS and who displayed no evidence of cancer activity or progression at the time of the GBS diagnosis. This left an initial total number of associated cancers of 10 (9.17\% of the cohort).

Considering current definitions of paraneoplastic syndrome [4], we excluded a further 2 patients who had IgG paraprotein myeloma and an additional 2 patients who had malignancy diagnosed $>2$ years after the GBS presentation. One had prostatic carcinoma and the other a basal cell carcinoma, both 4 years after GBS.

Consequently, a total of 6 patients (5.5\% of our total cohort) fulfilled requirements for inclusion as malignancy-associated GBS as per existing criteria for paraneoplastic syndrome [4]. Amongst these, 3 patients developed malignancy prior to the GBS presentation. All of them had developed GBS while undergoing treatment for their underlying cancer.

Chemotherapy was not implicated in the development of neuropathy in any of these 3 patients. Two patients were diagnosed with cancer during their GBS presentation. One patient developed cancer one year after the GBS diagnosis. Of the 6 patients whose GBS fulfilled criteria for paraneoplastic neurological syndrome, there was one case of angioimmunoblastic T-cell lymphoma, one of poorly differentiated squamous cell carcinoma of the nasal septum, one of gastric adenocarcinoma, one of hepatocellular carcinoma due to hepatitis B, one of rectal carcinoma with liver metastasis and one case of myelodysplastic syndrome. 
Considering U.K. cancer incidence rates [6], as well as the number of patient years of followup taking into account timing of death, the expected cumulative cancer rate in this GBS cohort of 109 patients over the 15-year study period, including the 3 years after admission of the last recruited patient (December 2015), was of 4.80 patients. With 10 cancer cases considered, the odds ratio was 2.08 (95\% CI: 1.06-3.71) and therefore significantly higher than expected $(\mathrm{p}=0.036)$. However, considering only the 6 cases meeting the definition for paraneoplastic syndrome [4], there was no increased risk of cancer in GBS patients over the study period (standardized odds ratio: $1.25 ; 95 \%$ CI: 0.51-2.60; $\mathrm{p}=0.559$ ).

We analyzed our results considering the 6 cancer cases fulfilling criteria for paraneoplastic syndrome. These are summarized in Table 1. Of the 6 patients meeting this definition, 5 were males and 1 was female as compared to 70 males and 33 females in the non-malignancy group ( $\mathrm{p}=0.66$ ). Average age in the malignancy group was higher compared to the nonmalignancy group (mean of 65.8 [S.D.:13.3] vs. 51.3 years [S.D.:17.5]; $p=0.043$ ). None of the patients in the malignancy group had positive anti-ganglioside antibodies or antineuronal antibodies (anti-Hu, Yo, Ri, CRMP5). There was no difference of lowest forced vital capacity (FVC) recorded among both groups $(\mathrm{p}=0.78)$. Cerebrospinal fluid (CSF) studies were acellular in all cases in both groups. CSF protein levels were significantly lower in patients with cancer than in those without cancer (mean: $0.34 \mathrm{~g} / \mathrm{dL}$ [S.D.: 0.59] vs. mean: $1.37 \mathrm{~g} / \mathrm{dL}$ [S.D.: 1.21]; $\mathrm{p}=0.002)$. Hyponatremia was significantly more common amongst patients with malignancy (66.7\% vs. $23.3 \%$; $\mathrm{p}=0.037)$. A higher proportion $(3 / 6 ; 50 \%)$ of the patients in the malignancy group required ICU admission with mechanical ventilation compared to of those from the non-malignancy group $(15 / 103 ; 14.6 \%)$, this approaching significance $(p=0.055)$. Average length of stay was comparable in both groups (mean of 42.8 days vs. 23.6 days; $\mathrm{p}=0.20$ ). Although admission MRCSS were comparable in malignancy and non- 
malignancy groups), improvement during in-patient stay, defined as amelioration of admission MRCSS (Medical Research Council Sum Score) was significantly less frequent in the malignancy group $(\mathrm{p}=0.023)$. From the point of view of electrophysiological subtypes, 3 patients had acute inflammatory demyelinating polyradiculoneuropathy (AIDP) and 3 had axonal GBS, as categorized by new electrodiagnostic criteria [7]. There was no difference in the number of patients with different subtypes of GBS in both groups ( $p=1$ for AIDP and $\mathrm{p}=0.66$ for axonal GBS). Summated compound muscle action potential (CMAP) was significantly lower in the malignancy group (mean: $14.5 \mathrm{mV}$, S.D.: 10.7 vs. mean: $7.1 \mathrm{mV}$, S.D.: 7.0; $\mathrm{p}=0.0497)$. There was no difference in terms of treatment given to patients in both groups with the majority (100\% and $91.3 \%$ from malignancy and non-malignancy groups, respectively), treated with intravenous immunoglobulins. Only 2 of 6 patients (33.3\%) with malignancy were able to walk at discharge, comparing to 73 of 103 patients (70.9\%) without malignancy ( $\mathrm{p}=0.075$ ). In-patient mortality was significantly higher in the malignancy group compared to the non-malignancy group $(33.3 \%$ vs. $1 \% ; \mathrm{p}=0.0075)$.

Considering all 10 cancer cases, the analysis produced similar statistical results with significance however not reached for more frequent hyponatremia in patients with malignancy $(\mathrm{p}=0.12)$ nor for MRC grade improvement during in-patient stay $(\mathrm{p}=0.10)$. 


\section{Discussion.}

The relationship between GBS and malignancy has been a topic of controversy since its initial descriptions. An earlier report described 2 patients with typical GBS occurring in association with Hodgkin's disease and oat-cell lung carcinoma, without evidence of malignant invasion [1]. The likelihood of cancer as a possible precipitating cause of GBS was raised, through an immune response towards the peripheral nervous system. Since then, many anecdotal reports relating GBS with various types of malignancy have been published. Cases described include malignancy of lung, bladder, blood, colon and skin $[2,8,9,10]$. One major difficulty with previous reports is that some cases appear clearly likely paraneoplastic from onset despite a GBS-like presentation, while others, differed with a classical clinical GBS presentation, with no associated features to suggest a paraneoplastic phenomenon, despite an eventually diagnosed associated malignancy, raising the question about a link between the 2. Our patients are not in the first case scenario as all were diagnosed with GBS and this remained the final diagnosis at discharge/death. The focus of our study has therefore been on the basis of the second eventuality, which is of direct relevance to clinical management of patients presenting with GBS.

Paraneoplastic neurological syndromes occur in less than $1 \%$ of patients with malignancy [3, 11]. Many cases present months to years before the diagnosis of cancer [3, 11]. Recent developments in concept of paraneoplastic syndrome has led to the inclusion of varieties of neuropathies other than the classical paraneoplastic syndromes. Criteria for diagnosis of paraneoplastic PNS disorder were adopted from Graus et al., 2004 [4]. Definite paraneoplastic disorders of PNS include a classic paraneoplastic syndrome associated with cancer and onconeural antibodies. Paraproteinemic neuropathies are not included as 
paraneoplastic manifestation, although exception to this rule may be made in the case of

POEMS syndrome, as it is defined [12]. GBS has been considered as one of the "nonclassical paraneoplastic neurological syndromes without onconeuronal antibodies" [13]. Since GBS is not a classical PNS and there are no identified onconeuronal antibodies linking it to a specific tumor, improvement of the neuropathy following treatment of the tumor is a major criterion for diagnosis confirmation. However, most cases would have been treated with immunotherapy during the course of illness making the definite diagnosis of paraneoplastic syndrome, impossible.

A single population-based study by Vigliani et al. has demonstrated a possible association between GBS and cancer [2]. However, the occurrence of GBS in this cohort of patients did not meet all the criteria for paraneoplastic syndrome, as were subsequently proposed by Graus et al. [4]. In this regard, Vigliani et al. included only malignancies developing or recurring 6 months before or after GBS diagnosis [2]. This excludes delayed cancer diagnoses, known to occur in some cases years after the neurological presentation. The definition used for the association is therefore of major importance in determining the existence of a link between the 2 disorders. We here used 2 definitions and confirm a variable risk, depending on which one is used. Although cancer in GBS, itself considered as a possible non-classical paraneoplastic syndrome [4] appears no more common than cancer in the general population, the total number of cancers does appear significantly higher in a cohort of patients with a GBS presentation. One reason may be that the definition for paraneoplastic neurological disease is too restrictive and wrongly excludes several relevant cases where there still may exist a causal relationship between the cancer and the neurological presentation, here GBS. 
The mechanisms of suspected paraneoplastic neuropathies without onconeuronal antibodies are unknown. Anti-ganglioside antibodies have been described in some of GBS patients with cancer, such as melanoma [14]. GBS encompasses a group of peripheral nerve disorders of autoimmune origin with, in axonal forms, evidence supporting the underlying pathophysiological mechanism involving the presence of molecular mimicry between gangliosides and antigens of an antecedent infection, stimulating a cross-reaction with peripheral nerve components [15]. Previous cases have been described of paraneoplastic motor neuropathy in patients with anti-GM1 ganglioside antibodies associated with epidermoid esophageal carcinoma [16], melanoma [10], bladder [8] and small cell lung carcinoma $[17,18]$. Whether the detection of anti-gangliosides antibodies may be relevant in patients with GBS with malignancy remains however uncertain. It was postulated that the expression of gangliosides in the neoplastic tissue may elicit autoimmune responses against neural structures [19]. In a case-control study comparing 29 patients with cancer and neuropathies with controls, anti-ganglioside antibodies, mainly IgM anti-GM1 were more frequently found in patients with cancer [20]. However, the pattern and levels of antibodies were not different from those of the controls and it appeared possible that the higher occurrence of anti-ganglioside antibodies in cancer patients may relate to a concurrent antitumor immune reaction rather than causative of the neuropathic process. The absence of antigangloside antibodies in all cases with cancer in our GBS cohort would similarly plead against their implication in the neuropathy. Despite absence of a definite antibody marker, it is possible that malignancy-induced immune dysregulation may be implicated in the pathogenesis of the acute inflammatory polyradiculoneuropathic process in patients with cancer and GBS. 
Our findings in relation to CSF protein, hyponatremia and electrophysiology are interesting. Elevated CSF protein levels are the expression of an inflammatory neural process, and it is possible that there may be pathophysiologic differences between the acute polyradiculoneuropathy of neoplastic disease and that of typical GBS explaining lower CSF protein in patients with malignancy. We unfortunately, in view of the retrospective nature of the analysis, do not however have the precise timings of lumbar punctures in many of our patients, some having been transferred from local district general hospitals to our center during their illness. We were therefore unable to ascertain whether patients with and without malignancy had lumbar punctures at comparable times. This is a drawback as early lumbar puncture may result in a higher likelihood of normal CSF protein levels. Hyponatremia was otherwise found more commonly in patients with malignancy as may have been expected, as not uncommon in cancer. In this regard, in view of the mild hyponatremia in the affected patients in the current analysis, further testing with serum and urine osmolality did not appear to have been performed. We previously found that hyponatremia is non-specific but a likely predictor of mortality in GBS [21]. Our current findings interestingly therefore suggest that hyponatremia may potentially increase suspicion of an underlying neoplasm in some patients with GBS. Finally, and similarly, more severe electrophysiological axonal loss was more frequent in patients affected by malignancy. Whether this may relate to associated preexisting asymptomatic neuropathy or to a more severe acute axonopathy, specifically occurring in these patients, is unknown.

In conclusion, our findings suggest that there may be an overall excess cancer risk in patients with a clinical diagnosis of GBS. Our results are in keeping with only the previous similar study performed [2]. The findings appear importantly also dependent on the definition used for cancer-associated GBS, and application of the strict published criteria for paraneoplastic 
syndrome reduced the number of cases and suggested absence of a link. We acknowledge the findings therefore need to be considered with caution. However, these results may suggest importantly for clinical practice, that consideration of an associated, detectable or occult malignancy is warranted in some patients with a clinical presentation of GBS. This is particularly relevant in absence of a preceding infectious trigger in older age groups, in patients who do not improve as would be expected after treatment, as well as those with normal CSF protein, hyponatremia, and severe electrophysiological axonal loss. Our results finally raise the issue of adequate investigations which in such cases may justify early use of whole-body CT imaging as well as PET. Furthermore, although the long-term monitoring of patients with GBS months to years after diagnosis is rare in clinical practice, this may require consideration with careful clinical re-assessment for adequate further investigations at regular intervals, in selected cases. 


\section{References.}

1. Klingon GH. The Guillain-Barré syndrome associated with cancer. Cancer 1965;18:157-63.

2. Vigliani MC, Magistrello M, Polo P, Mutani R, Chiò A. Risk of cancer in patients with Guillain-Barre syndrome (GBS). J Neurol 2004;251:321-326.

3. Rudnicki SA, Dalmau J. Paraneoplastic syndromes of the spinal cord, nerve, and muscle. Muscle Nerve 2000;23:1800-1818.

4. Graus F, Delattre JY, Antoine JC, Dalmau J, Giometto B, Grisold W, et al. Recommended diagnostic criteria for paraneoplastic neurological syndromes. J Neurol Neurosurg Psychiatry 2004;75:1135-1140.

5. Wakerley BR, Uncini A, Yuki N, GBS Classification Group. Guillain-Barré and Miller Fisher syndromes--new diagnostic classification. Nat. Rev. Neurol. 2014;10:537-544.

6. Cancer U.K. http://www.cancerresearchuk.org/health-professional/cancer-statistics July 2016.

7. Rajabally YA, Durand MC, Mitchell J, Orlikowski D, Nicolas G. Electrophysiological diagnosis of Guillain-Barré syndrome subtype: could a single study suffice? J. Neurol. Neurosurg. Psychiatry 2015;86:115-119.

8. Lagrange E, Veran O, Besson G. Pure motor relapsing Guillain-Barre's syndrome associated with anti-GM1 antibodies revealing urinary bladder cancer European Journal of Neurology 2007;17:e7.

9. Vatandoust S, Joshi R and Price TJ. Guillain-Barré syndrome in colorectal cancer. Asia-Pacific Journal of Clinical Oncology 2012;8:205-208.

10. Nicolae CD, Nicolae I. Antibodies against GM1 gangliosides associated with metastatic melanoma. Acta Dermatovenerol Croat. 2012;21:86-92. 
11. Darnell RB, Posner JB. Paraneoplastic syndromes involving the nervous system. N Engl J Med 2003;349:1543-1554.

12. Dispenzieri A. POEMS syndrome: update on diagnosis, risk-stratification, and management. Am J Hematol. 2015;90:951-962.

13. Rudnicki SA, Dalmau J. Paraneoplastic syndromes of the peripheral nerves. Curr Opin Neurol 2005;18:598-603.

14. Kloos L, Sillevis Smitt P, Ang CW, Kruit W, Stoter G. Paraneoplastic ophthalmoplegia and subacute motor axonal neuropathy associated with anti-GQ1b antibodies in a patient with malignant melanoma. J Neurol Neurosurg Psychiatry 2003;74:507-509.

15. Yuki N, Hartung HP. Guillain-Barré syndrome. N Engl J Med 2012;366:2294-2304.

16. Mostoufizadeh S, Souri M, de Seze J. A case of paraneoplastic demyelinating motor polyneuropathy. Case Rep Neurol 2012;4:71-76.

17. Watanuki S, Kinoshita K, Oda A, Kobayashi H, Satoh H, Tokuda Y. Occam's Razor or Hickam's dictum: a paraneoplastic or coincidental occurrence of lung cancer and Guillain-Barré syndrome. Inter Med 2014;53:1569-1573.

18. Itou T, Enomoto S, Makita Y, Enomoto H, Kuroda K, Kimura T, et al. A patient of sensorimotor neuropathy with small cell lung carcinoma and anti-GM1 antibody. Rinsho Shinkeigaku 2002;42:878-80.

19. De Toni L, Marconi S, Nardelli E, Alberti D, Borsellino G, Fracasso G, et al. Gangliosides act as onconeural antigens in paraneoplastic neuropathies. $\mathbf{J}$ Neuroimmunol 2004;156:178-187.

20. Antoine JC, Camdessanché JP, Ferraud K, Caudie C. Antiganglioside antibodies in paraneoplastic peripheral neuropathies. J Neurol Neurosurg Psychiatry. 2004;75:1765-1767. 
21. Hiew FL, Winer JB, Rajabally YA. Hyponatraemia in Guillain-Barré syndrome revisited. Acta Neurol Scand 2016;133:295-301. 
Table 1: Characteristics of 109 Guillain-Barré syndrome (GBS) patients, from Birmingham, U.K. (20012012) without and with malignancy as per paraneoplastic definitions [4].

\begin{tabular}{|l|l|l|l|}
\hline & GBS with malignancy & $\begin{array}{l}\text { GBS without } \\
\text { malignancy }\end{array}$ & $\begin{array}{l}\text { P value } \\
\text { (Fisher Exact } \\
\text { Test or T- } \\
\text { test) }\end{array}$ \\
\hline Number of patients & $6(5.5 \%)$ & $103(94.5 \%)$ & - \\
\hline Mean Age (SD; range) & $\begin{array}{l}65.8 \text { years }(13.3 ; 59- \\
87)\end{array}$ & 51.3 years (17.5) & $\mathbf{p}=\mathbf{0 . 0 4}$ \\
\hline Male: Female ratio & $5: 1$ & $70: 33$ & $\mathrm{p}=0.66$ \\
\hline AIDP Subtype & 3 & 47 & $\mathrm{p}=1$ \\
\hline Axonal GBS subtype & 3 & 36 & $\mathrm{p}=0.66$ \\
\hline Length of stay & 42.8 days & 23.6 days & $\mathrm{p}=0.20$ \\
\hline Hyponatremia & $4(66.7 \%)$ & $24(23.3 \%)$ & $\mathbf{p}=\mathbf{0 . 0 3 7}$ \\
\hline Average CSF protein mean (SD) & $0.34 \mathrm{~g} / \mathrm{L}(0.59)$ & $1.37 \mathrm{~g} / \mathrm{L}(1.21)$ & $\mathbf{p}=\mathbf{0 . 0 0 2}$ \\
\hline Lowest FVC & & & $\mathrm{p}$ \\
\hline ICU + ventilation & $2.61 \mathrm{I}$ & $2.87 \mathrm{I}$ & $\mathrm{p}=0.78$ \\
\hline Treated with immunoglobulins & $3(50 \%)$ & $15(14.6 \%)$ & $\mathrm{p}=0.055$ \\
\hline Average CMAP sum score & $6(100 \%)$ & $94(91.3 \%)$ & $\mathrm{p}=1$ \\
\hline $\begin{array}{l}\text { Number of patients with } \\
\text { Improvement in MRCSS }\end{array}$ & $7.1 \mathrm{mV}(7.0)$ & $14.5 \mathrm{mV} \mathrm{(10.7)}$ & $\mathbf{p}=\mathbf{0 . 0 4 9 7}$ \\
\hline $\begin{array}{l}\text { Number of patients able to walk at } \\
\text { discharge }\end{array}$ & 2 & 60 & $\mathbf{p}=\mathbf{0 . 0 2 3}$ \\
\hline In-patient mortality & $2(33.3 \%)$ & 73 & $\mathrm{p}=0.075$ \\
\hline
\end{tabular}

